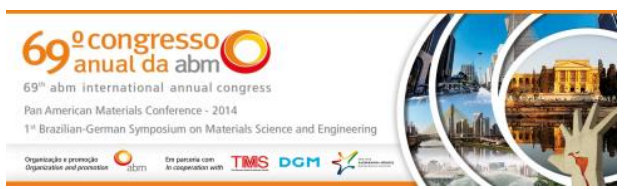

Tema: Soldagem

\title{
AVALIAÇÃO DAS PROPRIEDADES MECÂNICAS DE METAL DE SOLDA DE AÇO DE ALTA RESISTÊNCIA OBTIDO PELO PROCESSO DE SOLDAGEM GMAW PARA APLICAÇÃO EM COMPONENTES DE SISTEMAS DE ANCORAGEM*
}

\section{Resumo}

Amilton de Sousa Lins Junior ${ }^{1}$ Jorge Carlos Ferreira Jorge ${ }^{2}$ Luís Felipe Guimarães de Souza²

O presente trabalho faz parte de um amplo programa de pesquisas que envolvem o desenvolvimento de procedimentos de soldagem para componentes de sistemas de ancoragem de plataformas de petróleo. Particularmente, realiza-se um estudo das propriedades mecânicas de um metal de solda de alta resistência obtido pelo processo de soldagem GMAW para atendimento dos requisitos necessários para a soldagem de aços da classe IACS W22 Grau R4. Foram soldadas juntas multipasses pelo processo GMAW, com preaquecimento de $150^{\circ} \mathrm{C}$, corrente contínua, posição plana e aporte térmico médio de $1,0 \mathrm{~kJ} / \mathrm{mm}$. Após a soldagem, realizaram-se ensaios mecânicos e metalográficos em corpos-de-prova retirados integralmente do metal de solda depositado, tanto na condição de como soldado quanto após tratamento térmico pós-soldagem a $600^{\circ} \mathrm{C}$ por 1 hora. Os resultados mostraram que foi possível obter propriedades mecânicas que atendem os requisitos de aços da classe Grau R4 em todas as condições analisadas. Adicionalmente, verificou-se que o tratamento térmico pós-soldagem propiciou uma melhoria na tenacidade ao impacto devido ao revenimento da microestrutura do metal de solda.

Palavras-chave: Metal de solda; Propriedades mecânicas; Tratamento térmico.

\section{EVALUATION OF MECHANICAL PROPERTIES OF HIGH STRENGTH STEEL WELD METAL OBTAINED BY GMAW WELDING PROCESS FOR APPLICATION IN COMPONENTS OF MOORING SYSTEMS}

\section{Abstract}

The present work is part of a research program for the development of welding procedures for components of mooring systems of oil platforms. Particularly, high strength steel weld metal mechanical properties welded by GMAW process are discussed to meet the requirements of IACS W22 Grade R4 Rules. Welded joints by GMAW process were prepared, using a preheat of $150^{\circ} \mathrm{C}$, direct current, flat position and heat input of $1.0 \mathrm{~kJ} / \mathrm{mm}$. After welding, mechanical tests and metallographic examination were performed in specimens removed integrally from the weld metal, both in as welded and heat treated at $600^{\circ} \mathrm{C}$ conditions. The results shows that the obtained weld metals have mechanical properties higher than the minimum required for the welding of a IACS W22 R4 Grade steel. Additionally, the PWHT promoted an improvement on impact toughness due to the tempering of the microstructure.

Keywords: Weld metal; Mechanical properties; Post weld heat treatment.

1 Eng. Mecânico, M.Sc., Discente, PGMEC, UFF, Rio de Janeiro, RJ, Brasil.

2 Eng. Metalúrgico, M.Sc. DSc., Professor, PPEMM, CEFET-RJ, Rio de Janeiro, RJ, Brasil.

\footnotetext{
* Contribuição técnica ao 69 Congresso Anual da ABM - Internacional e ao 14 ENEMET - Encontro Nacional de Estudantes de Engenharia Metalúrgica, de Materiais e de Minas, 21 a 25 de julho de 2014, São Paulo, SP, Brasil.
} 


\section{INTRODUÇÃO}

$\mathrm{Na}$ utilização de estruturas soldadas, observa-se a importância crescente da necessidade de juntas soldadas com propriedades mecânicas adequadas às condições de serviço, cujas exigências podem inviabilizar um reparo por soldagem caso não apresente a confiabilidade exigida. Como exemplos de condições de serviço severas, são citadas as que cercam o emprego de plataformas marítimas e as desenvolvidas em regiões de baixas temperaturas [1]. No caso de operações offshore, a confiabilidade depende fundamentalmente da segurança das linhas de ancoragem. Além da necessidade de desenvolvimento tecnológico, ressalta-se a preocupação com a questão dos custos, cujos níveis têm levado fabricantes de componentes e estruturas marítimas a dedicarem atenção especial à elaboração de procedimentos de fabricação e reparo, apoiados em fundamentos técnico-científicos consistentes [2]. Estima-se que o valor da recuperação por soldagem de um equipamento danificado possa variar de $10 \%$ até $30 \%$ do valor de um novo. Outro aspecto relatado [3], diz respeito à necessidade da redução de peso de estruturas, fato que tem contribuído para o desenvolvimento de aços de alta resistência mecânica. Destaca-se ainda que os aços para esta aplicação [3] apresentam em comum o fato de possuírem baixo teor de carbono, característica que facilita a soldabilidade e propicia uma tenacidade satisfatória.

Para acompanhar o desenvolvimento dos aços de alta resistência, faz-se necessário um estudo paralelo de consumíveis de soldagem que permitam a soldagem destes aços. Neste aspecto, apresenta-se o presente trabalho como parte de um projeto de pesquisa mais amplo, no qual se avalia o comportamento de juntas soldadas em materiais aplicados em diversos equipamentos utilizados na ancoragem de navios, plataformas de petróleo e outros sistemas de operação flutuantes.

Dentro deste programa de pesquisa, foram desenvolvidos diversos estudos [4-25] sobre as propriedades mecânicas de juntas soldadas de equipamentos utilizados em linhas de ancoragem, com análise destas propriedades mesmo após ensaios de fadiga dos componentes $[9,11,13,21]$, os quais revelaram ser necessário uma análise criteriosa dos materiais envolvidos nesta aplicação, que apresentam requisitos severos de resistência e tenacidade, conforme mostrado na Tabela 1, que apresenta as exigências da International Association of Classification Societies (IACS) [26], que unificou as normas da Sociedades Classificadoras Navais, com destaque para o aço grau R4, por ser o de maior utilização atualmente.

A observação dos requisitos da Tabela 1, permite inferir que a tarefa de especificação de procedimentos de soldagem e de seleção de consumíveis para esta aplicação se torna muito complexa, notadamente em relação às propriedades do metal de solda, avaliada como a região crítica da junta soldada em estudos anteriores [5-7,11,27], visto que as normas de qualificação de consumíveis de soldagem [28] são limitadas à resistências mecânicas da ordem de $120 \mathrm{ksi}$ $(830 \mathrm{MPa})$, portanto inferiores ao valor de $860 \mathrm{MPa}$ especificado para o aço grau R4 e nem sempre definem critérios de resistência ao impacto mínima. Isto torna necessária uma seleção de consumível sem garantia de aprovação de norma. Adicionamente, as normas de aprovação não fazem menção à manutenção de propriedades após a realização de tratamento térmico de alívio de tensões, tratamento mandatório para acessórios de ancoragem soldado $[6,7]$.

Mesmo com todas estas limitações, a análise dos resultados do programa de pesquisa permitiu evidenciar ser possível obter metais de solda com propriedades

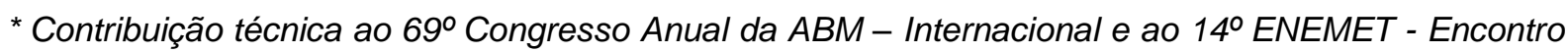
Nacional de Estudantes de Engenharia Metalúrgica, de Materiais e de Minas, 21 a 25 de julho de 2014, São Paulo, SP, Brasil.
} 


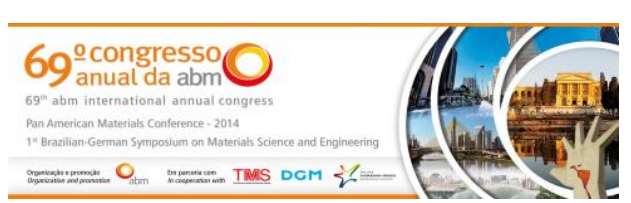

mecânicas que atendam os requisitos do aço grau $\mathrm{R} 4$ pelo processo eletrodo revestido [22,23].

Com base nestes bons resultados anteriores [22,23] e com o objetivo de promover uma melhoria da produtividade na operação da soldagem destes componentes de linhas de ancoragem, este trabalho realiza uma avaliação das propriedades mecânicas de metal de solda obtido pelo processo de soldagem GMAW e realiza um comparativo com as propriedades mecânicas obtidas anteriormente [22,23] pelos estudos onde fora empregado o processo de soldagem eletrodo revestido de forma a verificar a confiabilidade de utilização também deste processo na aplicação.

Tabela 1. Propriedades mecânicas dos aços segundo a norma IACS W22 [26].

\begin{tabular}{c|c|c|c|c|c}
\hline Grau do Aço & LE (MPa) & LR (MPa) & Al (\%) & RA (\%) & $E_{\mathrm{cv}}(\mathrm{J})$ \\
\hline R3 & 410 & 690 & 17 & 50 & 40 \\
\hline R3S & 490 & 770 & 15 & 50 & 45 \\
\hline R4 & 580 & 860 & 12 & 50 & 50 \\
\hline R4S & 700 & 960 & 12 & 50 & 56 \\
\hline R5 & 760 & 1000 & 12 & 50 & 58
\end{tabular}

Nota: LE- limite de escoamento; LR- limite de resistência; Al(\%)- alongamento percentual, RA(\%)- redução de área e $E_{C V^{-}}$energia absorvida Charpy- $V$ à $-20^{\circ} \mathrm{C}$.

\section{MATERIAIS E MÉTODOS}

\subsection{Materiais}

Utilizou-se para o estudo um arame sólido de 1,2mm de diâmetro, cuja composição química do metal depositado é apresentada na Tabela 2.

Tabela 2. Composição química do metal de solda.

\begin{tabular}{c|c|c|c|c|c|c|c|c|c|c|c|c}
\hline Elemento & $\mathrm{C}$ & $\mathrm{Si}$ & $\mathrm{P}$ & $\mathrm{S}$ & $\mathrm{Mn}$ & $\mathrm{Mo}$ & $\mathrm{Ni}$ & $\mathrm{Cr}$ & $\mathrm{Cu}$ & $\mathrm{V}$ & $\mathrm{Ti}$ & $\mathrm{Ceq}\left(^{*}\right)$ \\
\hline$\%$ Peso & 0,087 & 0,53 & 0,015 & 0,008 & 1,58 & 0,43 & 1,77 & 0,28 & 0,03 & 0,008 & 0,007 & 0,615 \\
\hline
\end{tabular}

\subsection{Soldagem dos Corpos-de-Prova}

As juntas foram soldadas a partir de chapas com $19 \mathrm{~mm}$ de espessura e $750 \mathrm{~mm}$ de comprimento. A geometria e demais dimensões da junta são apresentadas na Figura 1.

Utilizou-se uma temperatura de preaquecimento e de interpasse de $150^{\circ} \mathrm{C}$ e uma mistura de $\mathrm{Ar}-20 \% \mathrm{CO}_{2}$ como gás de proteção, sendo a vazão de $18 \mathrm{l} / \mathrm{min}$.

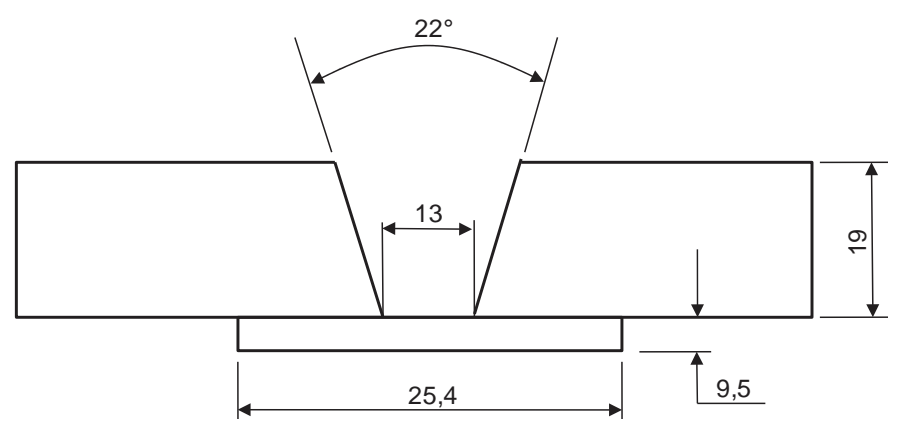

Figura 1. Detalhes da geometria da junta utilizada. Cotas em $\mathrm{mm}$.

* Contribuição técnica ao 69 Congresso Anual da ABM - Internacional e ao 14ํㅡㄹ ENEMET - Encontro Nacional de Estudantes de Engenharia Metalúrgica, de Materiais e de Minas, 21 a 25 de julho de 2014, São Paulo, SP, Brasil. 
A Tabela 2 apresenta os parâmetros médios utilizados para a soldagem.

Tabela 2. Parâmetros de soldagem utilizados.

\begin{tabular}{c|c|c|c|c}
\hline $\begin{array}{c}\Phi \\
(\mathrm{mm})\end{array}$ & $\begin{array}{c}\text { Corrente } \\
(\mathrm{A})\end{array}$ & $\begin{array}{c}\text { Tensão } \\
(\mathrm{V})\end{array}$ & $\begin{array}{c}\text { AT } \\
(\mathrm{kJ} / \mathrm{mm})\end{array}$ & $\begin{array}{c}\text { Número de } \\
\text { passes }\end{array}$ \\
\hline 1,2 & $220-236$ & $29-30$ & 1,0 & 19 \\
\hline
\end{tabular}

Nota: $\Phi$ - diâmetro do eletrodo; AT - aporte térmico.

\subsection{Tratamentos Térmicos Pós Soldagem (TTPS)}

Após a soldagem, foram realizados tratamentos térmicos consistindo de aquecimento a $600^{\circ} \mathrm{C}$ por 1 hora, sendo esta condição comparada à condição da junta de como soldada.

\subsection{Ensaios Mecânicos}

Foram removidos corpos-de-prova longitudinais e transversais ao cordão de solda para ensaios de tração, de impacto Charpy- $V$ e dureza. Os ensaios de tração foram realizados à temperatura ambiente, em corpos-de-prova retirados longitudinalmente ao cordão de solda, para avaliação da resistência mecânica do metal de solda. Os ensaios de impacto Charpy- $V$ foram realizados às temperaturas de $-40,-20$, e $0^{\circ} \mathrm{C}$, em corpos-de-prova normalizados $(10 \times 10 \times 55 \mathrm{~mm})$ e retirados transversalmente ao cordão de solda, a $2 \mathrm{~mm}$ da superfície da junta. $O$ entalhe foi posicionado no plano da espessura e no centro do cordão de solda. Foram realizados ensaios de dureza Vickers em corpos de prova transversais ao cordão de solda, sendo realizada uma varredura de dureza com aplicação de carga de $1 \mathrm{kgf}$ da superfície até a raiz do metal de solda.

\subsection{Ensaios Metalográficos}

Foram realizados ensaios macro e micrográficos por microscopia eletrônica de varredura (MEV) nos metais de solda, para avaliação das microestruturas.

Foram observados os constituintes microestruturais nas regiões correspondentes ao último passe (UP) e regiões colunar $(R C)$ e reaquecida $(R R)$ na ponta do entalhe Charpy-V.

A preparação para análise consistiu de lixamento e polimento com pasta de diamante com granulometria de 6,3 e $1 \mu \mathrm{m}$, seguido de ataque químico com Nital $2 \%$.

Adicionalmente, foi realizada a análise quantitativa do percentual de regiões colunar e reaquecida na ponta do entalhe Charpy- $V$.

\section{RESULTADOS E DISCUSSÃO}

\subsection{Propriedades Mecânicas e Microestruturais}

A Tabela 3 mostra os resultados dos ensaios de tração, realizados nos metais de solda, onde se nota que todos os resultados apresentam-se superiores aos mínimos exigidos para o aço grau R4 [26], mesmo considerando a redução provocada pelo TTPS.

O mesmo efeito é verificado nos resultados do ensaios de dureza mostrados na Figura 2.

* Contribuição técnica ao 69 Congresso Anual da ABM - Internacional e ao 14ํㅡㄹ ENEMET - Encontro Nacional de Estudantes de Engenharia Metalúrgica, de Materiais e de Minas, 21 a 25 de julho de 2014, São Paulo, SP, Brasil. 
Tabela 3. Resultados dos ensaios de tração dos metais de solda.

\begin{tabular}{c|c|c|c|c}
\hline Condição & LE(MPa) & LR(MPa) & $\mathrm{Al}(\%)$ & $\mathrm{RA}(\%)$ \\
\hline Como Soldado & 939 & 962 & 13 & 56 \\
\hline TTPS & 829 & 881 & 16 & 57 \\
\hline Mínimo [26] & 580 & 860 & 12 & 50 \\
\hline
\end{tabular}

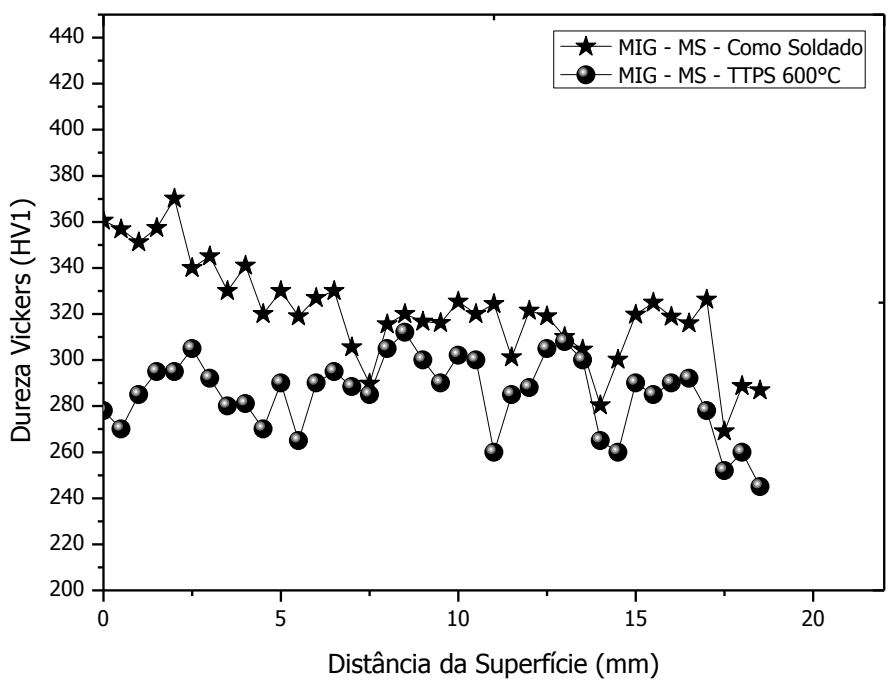

Figura 2. Resultados dos ensaios de dureza Vickers (HV1).

Isto é um fato extremamente relevante, já que como abordado pela literatura [30,31], a obtenção de valores elevados de resistência mecânica para os consumíveis de maior resistência tem sido o foco de maior preocupação, sendo apontado como o maior desafio a ser superado [10,14,15,17,30-32]. "Segundo Ramirez [30], Surian et al. [31] e Talas [33], uma forma de se ter uma estimativa da resistência mecânica do metal de solda de alta resistência é através do controle do carbono equivalente deste metal de solda". No entanto, esta afirmação deve ser vista apenas do ponto de vista qualitativo quando se opera com metais de solda de alta resistência, dado o elevado grau de dispersão de resultados, em função do efeito interativo dos diversos elementos de liga atuantes ainda não completamente entendidos. De fato, na Figura 3 mostra uma coletânea de resultados de diversos autores $[14,15,17,29,31,34]$, onde se verifica que os resultados obtidos no presente trabalho não apresentam boa correlação com estes dados experimentais, mesmo considerando a dispersão acentuada existente para maiores valores de carbono equivalente.

Este elevado grau de dispersão, pode estar associado com modificações microestruturais significativas. De fato, "segundo Ramirez [35], para valores de carbono equivalente superiores a $0,47 \%$, começa a predominar produtos de baixa temperatura de transformação, incluindo a martensita". Por outro lado, "Lord et al. [34] afirmam que para a faixa de velocidades de resfriamento normais de soldagem, não há temperabilidade suficiente para formação somente de martensita, sendo a microestrutura do metal de solda de alta resistência constituída de martensita e bainita". Associado a estas questões, deve-se ressaltar o fato que os corpos-deprova para ensaio de tração apresentam um diâmetro de $10 \mathrm{~mm}$ e, portanto,

* Contribuição técnica ao $69^{\circ}$ Congresso Anual da ABM - Internacional e ao 14ํㅡㄹ ENEMET - Encontro Nacional de Estudantes de Engenharia Metalúrgica, de Materiais e de Minas, 21 a 25 de julho de 2014, São Paulo, SP, Brasil. 


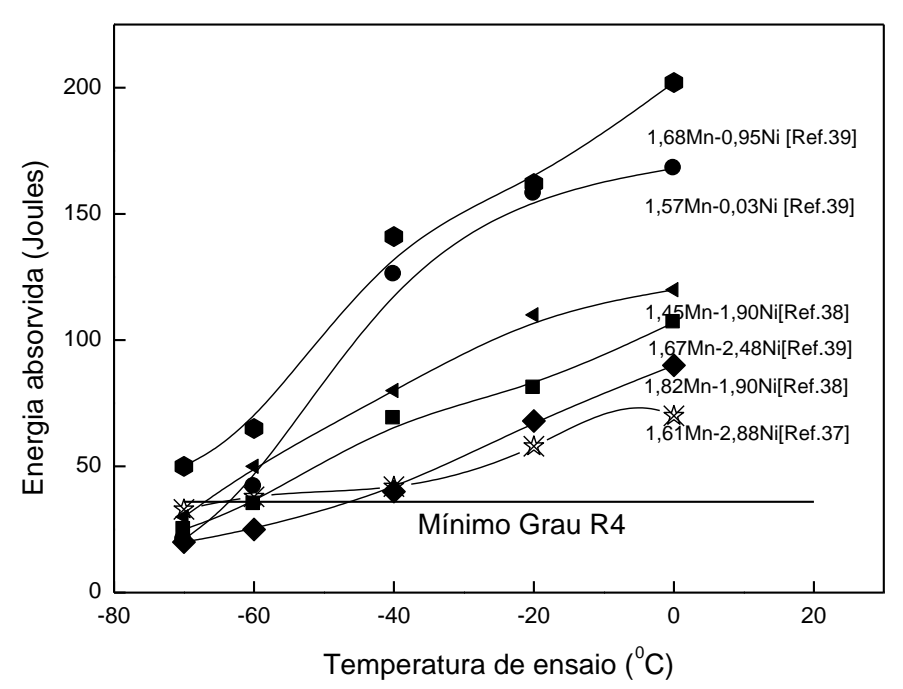

Figura 6. Influência da relação Ni-Mn na tenacidade ao impacto [37-39].

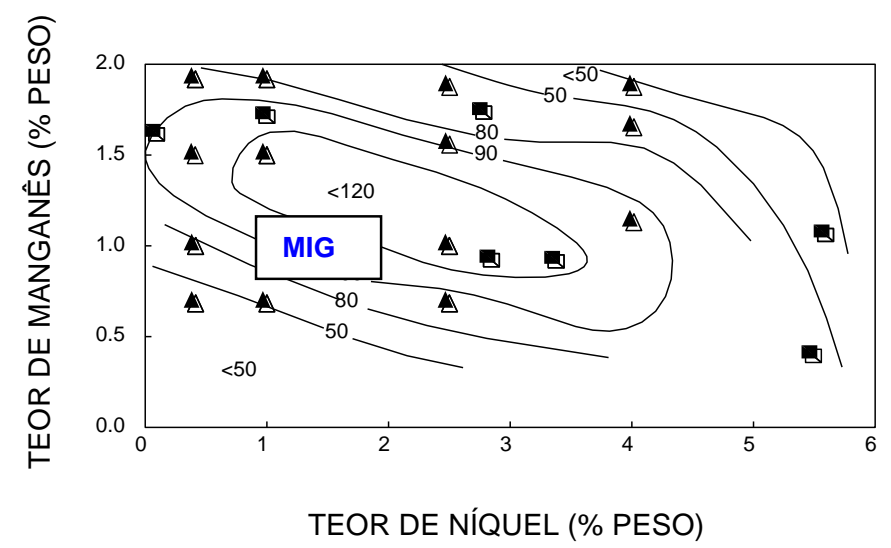

Figura 7. Efeito do níquel e manganês na tenacidade ao impacto à $-50^{\circ} \mathrm{C}$ [37].

A Figura 8 mostra os resultados dos ensaios de impacto realizados no metal de solda estudado, onde se nota que os valores de energia absorvida foram muito superiores ao mínimo exigido de 36 joules para o metal de solda [26] em todas as condições de análise mesmo para a temperatura de $-40^{\circ} \mathrm{C}$. De maior importância para o escopo do presente trabalho, foi notar que o efeito do TTPS provocou um aumento da tenacidade ao impacto, o que é atribuído ao revenimento da microestrutura (Figura 5). De fato, este é um resultado representativo, pois vários estudos realizados no programa de pesquisa, apontaram uma queda da tenacidade ao impacto do metal de solda após o TTPS, o que foi atribuído à precipitação de carbetos nos contornos de grão [10,12,15-20,23-25] , o que não ocorreu no caso presente.

\footnotetext{
* Contribuição técnica ao $69^{\circ}$ Congresso Anual da ABM - Internacional e ao 14ํㅡㄹ ENEMET - Encontro Nacional de Estudantes de Engenharia Metalúrgica, de Materiais e de Minas, 21 a 25 de julho de 2014, São Paulo, SP, Brasil.
} 

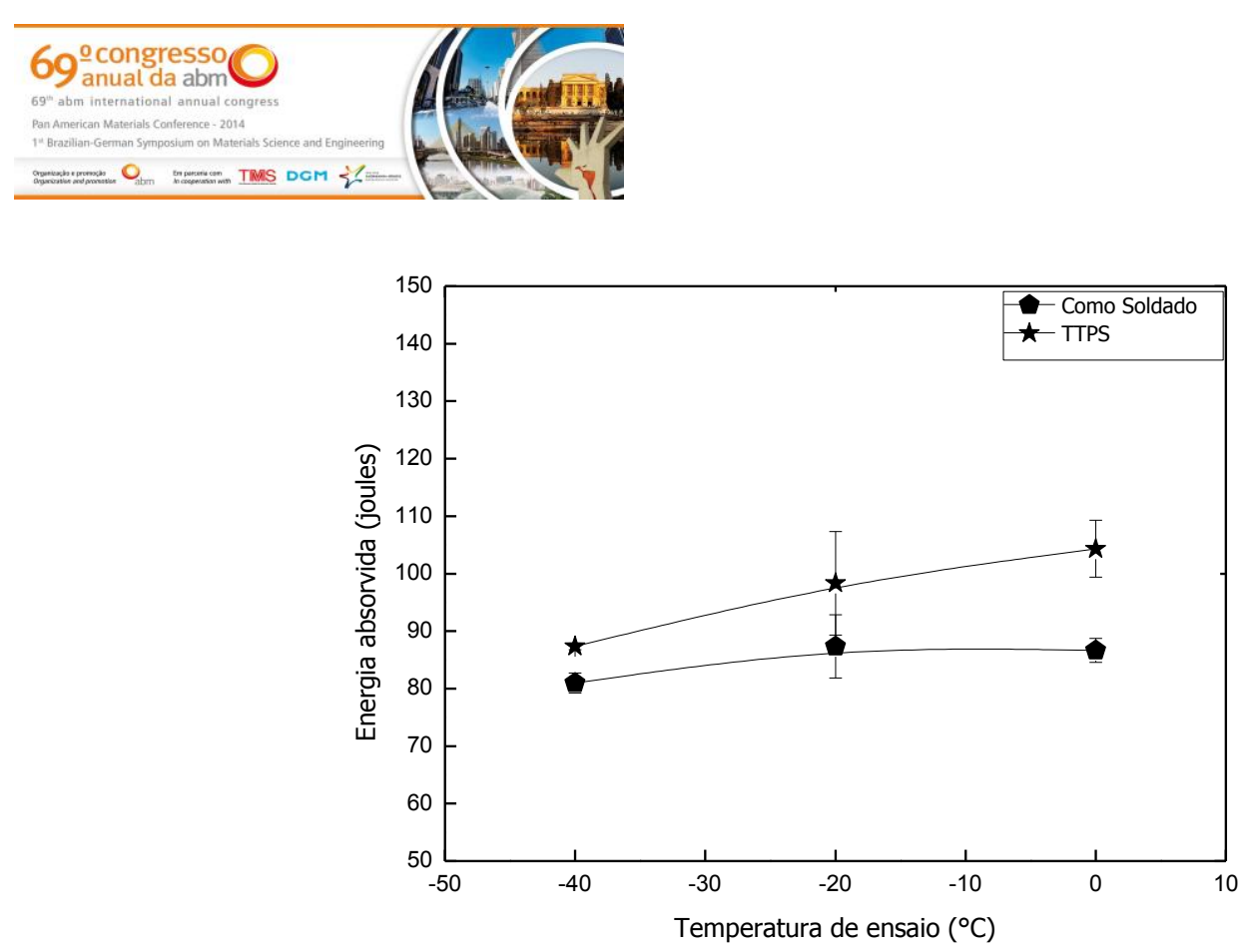

Figura 8. Variação da energia absorvida com a temperatura de ensaio.

Segundo "Keehan et al. [41], alta resistência e boa tenacidade podem ser obtidos em metais de solda para tempos de resfriamento entre $800^{\circ} \mathrm{C}$ e $500^{\circ} \mathrm{C}(\Delta t 8 / 5)$ entre 3 e 13 segundos, devido à microestrutura existente". Isto está de acordo com os resultados do presente trabalho, pois foi possível obter uma microestrutura constituída predominantemente por martensita revenida de baixo carbono (Figura 5).

\subsection{Avaliação do Consumível}

A Tabela 3 mostra os resultados dos ensaios de tração, realizados nos metais de solda, onde se nota que todos os resultados apresentam-se superiores aos mínimos exigidos para o aço grau R4 [26], mesmo considerando a redução provocada pelo TTPS. No entanto, cabe destacar que o resultado obtido após o TTPS, condição de utilização de juntas soldadas de projeto de componentes de ancoragem [6,7] é apenas $2 \%$ superior ao mínimo exigido para o aço grau $R 4$, o que poderia não garantir uma confiabilidade de aplicação. No entanto, existe uma questão importante a comentar que envolve as propriedades em condições reais de utilização são sempre afetadas pela diluição com o metal base. Desta forma, pode-se inferir que o resultado de resistência mecânica do presente trabalho seja conservador, em função da composição química normalmente utilizada nos materiais base de componentes de ancoragem.

Nesta mesma linha de avaliação, a tenacidade ao impacto do metal de solda estudado apresenta um comportamento com confiabilidade para utilização na aplicação em questão, visto os elevados valores de energia absorvida obtidos no estudo realizados.

Finalmente, é importante comentar que além de propiciar propriedades mecânicas satisfatórias, a soldagem pelo processo GMAW permite uma redução aproximada de 2,5 vezes no tempo de soldagem se comparada com o procedimento de união utilizado o processo com eletrodo revestido.

Considerando as evidências do presente trabalho, o consumível em estudo é recomendado para a soldagem de um aço grau $\mathrm{R} 4$, visando avaliar as propriedades da junta soldada com este consumível.

* Contribuição técnica ao 69ํㅡㄹ Congresso Anual da ABM - Internacional e ao 14º ENEMET - Encontro Nacional de Estudantes de Engenharia Metalúrgica, de Materiais e de Minas, 21 a 25 de julho de 2014, São Paulo, SP, Brasil. 


\section{CONCLUSÃO}

Do exposto no transcurso do presente trabalho, pode-se concluir que:

a) O consumível estudado, soldado com preaquecimento e temperatura interpasses de $150^{\circ} \mathrm{C}$, atingiu os requisitos do aço grau R4 para todas as condições de estudo;

b) O TTPS propiciou uma redução na resistência mecânica e aumento na tenacidade devido ao revenimento da microestrutura;

c) O consumível em estudo deve ser testado em junta de aço grau R4 para avaliar confiabilidade da resistência mecânica da junta soldada.

\section{Agradecimentos}

Os autores agradecem às Instituições pelo apoio prestado na execução do presente trabalho: A Fluke Engenharia Ltda., CEFET-RJ, ESAB, CNPq e FINEP.

\section{REFERÊNCIAS}

1 Jorge JCF, Rebello JMA. Influence of welding procedure on the microstructure and toughness of high strength ferritic steel weld metals, Revue de la Soudure, 2000; 56(1):4-12.

2 Mosciaro HB, Jorge JCF. Propriedades mecânicas de reparos por soldagem de aço fundido de alta resistência, Anais do XXI Encontro Nacional de Tecnologia da Soldagem, 1995, Caxias do Sul, 307-317.

3 Salvador LS, Jorge JCF, Bott IS. Efeito da composição química e tratamento térmico de alívio de tensões nas propriedades de metais de solda de alta resistência para aplicação em estruturas offshore, Anais do XXI Encontro Nacional de Tecnologia da Soldagem, 1995, Caxias do Sul, $337-351$.

4 Pacheco PMCL, Kenedi PP, Jorge JCF, Vieira RD. Residual stress analysis of studless chain links repaired by welding, Anais do $27^{\circ}$ Seminário de Inspeção de Equipamentos, 2001, Rio de Janeiro, 1-7.

5 Jorge JCF et al. Desenvolvimento de procedimento de reparo por soldagem de amarras de aço para ancoragem de plataformas de petróleo, Anais do XXVII Congresso Nacional de Soldagem , 2001, Campinas, 1-10.

6 Jorge JCF et al., Desenvolvimento de procedimento de reparo por soldagem de amarras de aço para ancoragem de plataformas de petróleo - Parte II - Homologação do Procedimento, Anais do XXVIII Congresso Nacional de Soldagem , 2002; São Paulo, 1-10.

7 Suman JA, Jorge JCF, Souza LFG, Bott IS. Efeito de tratamentos térmicos póssoldagem nas propriedades de aço fundido de elevada resistência para sistemas de ancoragem de plataformas marítimas, Soldagem \& Inspeção, 2004; 9(4):205-212.

8 Jorge JCF. Relação tenacidade/microestrutura da ZTA de aço fundido ASTM A 148 GR. 8050 para acessórios de ancoragem de plataformas de petróleo, Soldagem \& Inspeção, 2004; 9(4):192-197.

9 Jorge JCF et al. Desenvolvimento de procedimento de reparo por soldagem de amarras de aço para ancoragem de plataformas de petróleo, Parte III - comportamento em fadiga, Anais do XXXII Congresso Nacional de Soldagem, 2006; Belo Horizonte, 110.

10 Jorge JCF, Souza LFG, Santos Filho OR, Santos Filho AMF, Bott IS. Influência da composição química e tratamento térmico pós-soldagem nas propriedades mecânicas e microestruturais de metais de solda de alta resistência, Anais do XXXIII Congresso Nacional de Soldagem, 2007;1-10.

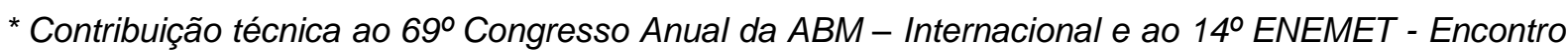
Nacional de Estudantes de Engenharia Metalúrgica, de Materiais e de Minas, 21 a 25 de julho de 2014, São Paulo, SP, Brasil.
} 


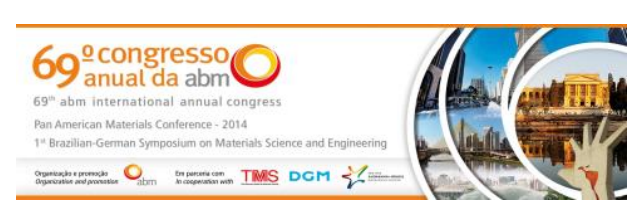

11 Jorge JCF, Souza LFG, Pacheco PMCL, Santos Filho AMF, Santos Filho OR, Diniz JLC, Bott IS. Evaluation of the mechanical properties on welded links of high strength steel mooring chains after fatigue testing, Proc. Of the 2nd Latin American Welding Congress, 2008;São Paulo, 1-10.

12 Farneze HN, Jorge JCF, Souza LFG, Bott IS. Estudo comparativo de metais de solda de aço de alta resistência obtidos pelos processos eletrodo revestido e arame tubular para aplicação em equipamentos de amarração offshore; Soldagem \& Inspeção, 2009;14(2):151-160.

13 Jorge JCF, Souza LFG, Pacheco PMCL, Kenedi PP, Santos Filho OR, Diniz JLC. Avaliação de propriedades mecânicas de elos de amarras de aço de alta resistência submetidos à ensaios de fadiga, Anais do VI Congresso Nacional de Engenharia Mecânica, 2010;Campina Grande, 1-10.

14 Faragasso SM, Souza LFG, Bott IS, Jorge JCF. Avaliação de propriedades mecânicas e microestruturais de metal de solda de aço de extra alta resistência para utilização em equipamentos de ancoragem, Anais do XXXVII CONSOLDA, 2011; Natal, 1-10.

15 Jorge JCF, Souza LFG, Santos Filho OR, Bott IS. Estudo de metais de solda de aço de extra alta resistência para utilização em componentes de ancoragem de plataformas de petróleo. Parte I: Propriedades Mecânicas, Anais do XXXVII CONSOLDA, 2011; Natal, 1-10.

16 Vogas PVBD, Jorge JCF, Souza LFG, Bott IS. Efeito do tratamento térmico póssoldagem nas propriedades de metal de solda de alta resistência obtidos por arame tubular tipo flux cored, Anais do $67^{\circ}$ Congresso Internacional da ABM, 2012; Rio de Janeiro, 374-387.

17 Gomes AJM, Jorge JCF, Souza LFG, Bott IS. Estudo comparativo de metais de solda de aços de extra alta resistência para utilização em componentes de linhas de ancoragem de plataformas de petróleo, Anais do $67^{\circ}$ Congresso Internacional da ABM, 2012, Rio de Janeiro, 806-818.

18 Pinheiro MM, Jorge JCF, Souza LFG, Bott IS. Efeito do tratamento térmico póssoldagem nas propriedades de metal de solda de alta resistência obtidos por arame tubular tipo metal cored, Anais do $67^{\circ}$ Congresso Internacional da ABM, 2012, Rio de Janeiro, 1323-1333.

19 Gomes AJM, Souza LFG, Bott IS, Jorge JCF. Estudo de propriedades mecânicas e microestruturais de metal de solda de extra alta resistência com diferentes tratamentos térmicos pós-soldagem, Anais do XXXVIII CONSOLDA, 2012, Ouro Preto, 1-13.

20 Vogas PVBD, Jorge JCF, Souza LFG, Bott IS. Influência do preaquecimento e tratamento térmico pós-soldagem nas propriedades de metal de solda de alta resistência obtido pelo processo arame tubular, Anais do XXXVIII CONSOLDA, 2012, Ouro Preto, 1-12.

21 Jorge JCF, Souza LFG, Pacheco PMCL, Kenedi PP, Santos Filho OR, Diniz JLC. Performance evaluation of high strength steel chain links welded by SMAW process submitted to fatigue testing, Anais do $6^{0}$ Congresso Nacional de Engenharia Mecânica, 2012, São Luis, 1-9.

22 Jorge JCF, Faragasso SM, Souza LFG, Bott IS, Efeito do tratamento térmico póssoldagem nas propriedades mecânicas e microestruturais de metal de solda de aço de extra alta resistência para utilização em equipamentos de ancoragem, Soldagem e Inspeção, 2013; 18(2):137-148.

23 Gomes AJM, Jorge JCF, Souza LFG, Bott IS. Influence of chemical composition and post welding heat treatment on the microstructure and mechanical properties of high strength steel weld metals. Materials Science Forum; 2013;758:21-32.

24 Gomes AJM, Jorge JCF, Souza LFG, Bott IS. Propriedades mecânicas de metal de solda de aço de extra alta resistência para reparos de componentes de amarração de plataformas, Anais do $7^{0}$ Congresso Brasileiro de Engenharia de Fabricação, 2013, Penedo, 1-11.

25 Pinheiro MM, Jorge JCF Souza LFG Bott IS. Efeito do preaquecimento e tratamento térmico pós-soldagem nas propriedades de metal de solda de alta resistência obtidos

\footnotetext{
* Contribuição técnica ao $69^{\circ}$ Congresso Anual da ABM - Internacional e ao 14ํㅡㄹ ENEMET - Encontro Nacional de Estudantes de Engenharia Metalúrgica, de Materiais e de Minas, 21 a 25 de julho de 2014, São Paulo, SP, Brasil.
} 
por arame tubular tipo metal cored, Anais do $7^{0}$ Congresso Brasileiro de Engenharia de Fabricação, 2013, Penedo, 1-11.

26 International Association of Classification Societies, W22, Offshore Mooring Chain, June, 2011.

27 Santos Filho OR, Jorge JCF. Alternative procedure for the replacement of chain links by manual welding - An overview, Prceedings of the International Mooring Seminar, 2012, Macaé, 1-39.

28 AWS 5.5, Specification for low alloy steel electrodes for shielded metal arc welding, 1996.

29 American Bureau of Shipping, Rules for Materials and Welding, Part 2, USA, 2012.

30 Ramirez JE, Examining the Mechanical Properties of High-Strength Steel Weld Metals, Welding Journal, 2009; 88(1): 32-38.

31 Surian E, Rissone NM, Svoboda HG, Vedia LA. SMAW, FCAW and SAW High-Strength Ferritic Deposits: The Challenge Is Tensile Properties, Welding Journal, 2010; 89(3): 54-64s.

32 Surian ES, de Vedia LA. All-weld-metal design for AWS E10018M, 11018M and 12018M type electrodes, Welding Journal, 1999; 78(6): 217s-228s.

33 Talas $\mathrm{S}$. The assessment of carbon equivalent formulas in predicting the properties of steel weld metals, Materials \& Design, 2010; 31:2649-2653.

34 Lord M, Jennings G. Effect of interpass temperature on properties of high-strength weld metals, Svetsaren, 1999; 54(1-2) 53-58.

35 Ramirez JE. Characterization of high strength steel weld metals, chemical composition, microstructure and nonmetallic inclusions, Welding Journal, 2008;87(3):65s-75s .

36 EN 1011-2, Welding - Recommendations for welding of metallic materials - Part 2: Arc welding of ferritic steels, December, 2003.

37 Zhang Z, Farrar RA. Influence of $\mathrm{Mn}$ and $\mathrm{Ni}$ on the Microstructure and Toughness of $\mathrm{C}$ Mn-Ni Weld Metals, Welding Journal, 1997; 76(5):183-196.

38 Surian E, Trotti J, Cassanelli AN, de Vedia LA, Influence of Mn content on mechanical properties and microstructure of a high strength SMA electrode weld metal, 1987, IIWIIS Doc. II-A-724-87.

39 Kang BY, Kim HJ, Hwang SK, Effect of Mn and Ni on the variation of the microstructure and mechanical properties of low-carbon weld metals, ISIJ International, 2000; 40(12): 1237-1245.

40 Sousa Junior AL, Jorge JCF, Souza LFG. Estudo comparativo das propriedades mecânicas de juntas soldadas pelos processos eletrodo revestido e MIG de aço de elevada resistência mecânica para emprego em estruturas naval, Anais do 7ํㅡㄹ Congresso Brasileiro de Engenharia de Fabricação, 2013, Penedo, p.1-10.

41 Keehan E, Zachrisson J, Karlsson L. Influence of cooling rate on microstructure and properties of high strength steel weld metal, Science and Technology of Welding and Joining, 2010;15(3): 233-238.

* Contribuição técnica ao 69 Congresso Anual da ABM - Internacional e ao 14ํㅡㄹ ENEMET - Encontro Nacional de Estudantes de Engenharia Metalúrgica, de Materiais e de Minas, 21 a 25 de julho de 2014, São Paulo, SP, Brasil. 